\title{
Communication
}

\section{Locate Objects Mechanical Damage Based on Fiber-Optic Communication Systems}

\author{
Rakhimov Baxtiyorjon Ne'matovich, Alimjonov Botirjon Abdulmaqsud O'g'li \\ Department of Electronics and Radio engineering, Tashkent University of Information Technologies Named After Muhammad al-Khwarizmi, \\ Tashkent, Uzbekistan
}

Email address:

Sardorjabbarov.sj@gmail.com (A. B. A. o'g'li)

To cite this article:

Rakhimov Baxtiyorjon Ne'matovich, Alimjonov Botirjon Abdulmaqsud O'g'li. Locate Objects Mechanical Damage Based on Fiber-Optic Communication Systems. Bioprocess Engineering. Vol. 2, No. 1, 2018, pp. 11-14. doi: 10.11648/j.ep.20180201.13

Received: February 28, 2018; Accepted: March 26, 2018; Published: May 7, 2018

\begin{abstract}
This work is devoted to the development of a method for monitoring the stability of hydraulic structures (dams), which created a monitoring system, operator can make a conclusion about the reliability of controlled structures as well as data on the level of protection facilities and experimental results are presented.
\end{abstract}

Keywords: Optical Fiber (OF), Light Detector, Photoelectric Signal Processing Unit (PSPU)

\section{Introduction}

Currently, optical fiber is widely used in fiber-optic communication systems, however, the quality of the fiber depends strongly signaling bandwidth capacity. Existing methods and devices for mechanical damage, disorders, defects do not allow a sufficient accuracy to identify such disadvantages as a change in signal characteristics of the inverter output photocurrents receiving diodes amplifier due to the different characteristics of the emitters and receivers, the difference in the optical coordination receivers and emitters fiber etc. [1].

Unlike other methods (acoustic, strain and so on. etc.) Optoelectronic systems using optical fibers (SC) allow control of complex design, VS can be embedded into the concrete products. The advantage of developing a monitoring method using VS is high accuracy detection of mechanical extensions at the same time control is carried out online.

The purpose of the work is to develop optimal conditions for the detection of mechanical damage to various structures (dams, bridges, civil construction) using optical fiber. Using the method of application of interferometric measurements, worked through the monitoring of mechanical structures for reliability and safety.

\section{Main Part}

Optical fibers can withstand relatively high operating temperatures and pressures - two important parameters that are decisive for the manufacture of a number of structures made of composite materials technology. Moreover, the fibers are relatively insensitive (or can be made relatively insensitive, if using certain techniques stacking) influences the corrosion and fatigue loads. The signal passing through the optical fiber, essentially insensitive to detrimental electromagnetic influences. Accordingly, there is no need for expensive and bulky electromagnetic shielding even in places where there are strong electromagnetic radiation sources (e.g., power plants). Optical fibers have a sufficiently wide signal bandwidth, which allows their use in a large number of applications. They also can simultaneously perform the function of the sensor signal and the measurement conductor (fiber optic data channels). Applying the method of multiplexing multiple sensors can be placed in one optical fiber, (such as: 2.5).

Existing diagnostic methods to control the stability of hydraulic structures do not allow to accurately determine these changes.

Developed a stand allows to work fairly accurate diagnostic method sustainability and reliability of hydraulic structures under the influence of external factors such as the strain (tension, compression), movement, cracking, pre-destruction.

One of perspective applications in the VS monitoring building structures can be considered to obtain the criteria by which an operator to create a monitoring system can draw a 
conclusion about the reliability of the controlled design, and, consequently, on the level of security.

The problem is solved in that the device comprises a sensor of the optical fibers, light emitting diodes, connected via current controller resistors to the output of the power amplifier photodetectors block photoelectric signal processor connected to the computer, an oscillator, a trigger, a second power amplifier asymmetrical connectors, the output of The master oscillator is coupled to an input of the flip-flop, the outputs of which are connected to the power amplifier input, light emitting diodes are interconnected in opposition and optically coupled are connected to the first and third asymmetrical couplers, the outputs of the second and fourth asymmetric optical connection connectors are connected to the successively interconnected photodetectors, the first outlet the first unbalanced coupler connected to the input of the first optical fiber sensor, a second end and the input of the second optical fiber sensor with a gap there between are fixedly attached to a solid structure, the output of the second optical fiber sensor is connected to a first input of a second asymmetric coupler, a second input of which the third optical waveguide sensor is connected to the second output third asymmetric coupler, the first output of which is the fourth optical waveguide sensor is connected to a second input of the fourth asymmetric coupler, the first input of which the sensor fifth waveguide connected to the second output of the first unbalanced coupler connection point of light emitting diodes connected to one of the terminals of the second photodetector, one terminal of the first photodetector is connected to power source and photodetectors connection point is connected to the input of the photoelectric signal processing unit (such as. 5).

Improved strain measurement accuracy is achieved in that the device is provided with a master oscillator, a trigger, the second power amplifier, nonsymmetric connectors, the output of the master oscillator is coupled to an input of the flip-flop, the outputs of which are connected to the power amplifier input, light emitting diodes are interconnected in opposition and optically coupled connected rigidly fixed to the first and third asymmetrical couplers, the outputs of the second and fourth asymmetric optical connection connectors are connected to the successively interconnected photodetectors, the first output of the first unbalanced coupler connected to the input of the first encoder LEDs second end and the input of the second sensor LED with a gap between them solid construction, output of the second optical fiber sensor is connected to a first input of a second asymmetric coupler, a second input of which the third optical waveguide sensor connected to the second output of the third asymmetric coupler, the first output of which transducer the fourth optical waveguide connected to the second input of the fourth asymmetric coupler, the first input of which the sensor fifth waveguide connected the second output of the first unbalanced coupler light-emitting diode connection point is connected to one of the terminals of the second photodetector, one terminal of the first photodetector is connected to a power source, and photodetectors connected to the connection point of entrance photoelectric signal processing unit.

Simplification of the construction is achieved by providing the oscillator device, trigger the second power amplifier and the asymmetrical connectors, reducing the number of elements compared to the closest analogue, simplify processing photoelectric signals from the photodetectors and, in addition, reduced power consumption, the sensor of the optical fibers easier attach to a solid structure. Figure 1 shows a block diagram for diagnosis of pre-fracture and deformation of solid structures.

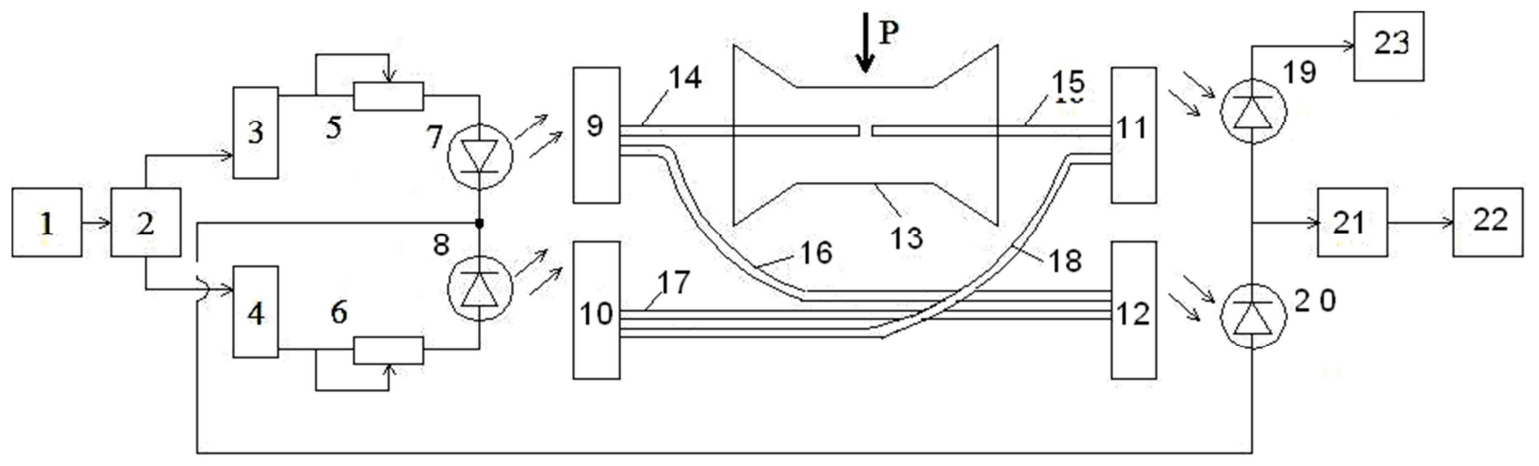

Figure 1. The device for the diagnosis of pre-fracture and deformation of solid structures.

The master oscillator 1; Trigger -2; power amplifiers - 3. 4; tokoreguliruyuschie registory - 5. 6; light-emitting diodes 7. 8, nesimmitrichnye connectors - 9, 10, 11, 12; solid-state design, 13; Sensor fibers - 14, 15, 16, 17, 18; photodetectors - 19.20; photoelectric signal processing unit - 21,; computer - 22; Power Supply 23.

The device is a two channel - measuring and reference.

Measuring channel includes a first amplifier 3, a first thermostatic resistor 5 , the light emitting diode 7 , the first and second asymmetric couplers 9. 11, 14. 15 fibers sensor photodetector 19 .

Reference channel includes a second power amplifier 4, the second resistor tokoreguliruyuschy 6 , the light emitting diode
8 , the third and fourth asymmetrical connectors $10,12,16,17$, 18 fibers sensor photodetector 20 .

The device operates as follows.

The master oscillator 1 generates rectangular pulses of repetition frequency fof $10 \mathrm{kHz}$, which is input to the flip-flop 2 and launch it into operation. With two opposite-O 2 trigger pulses separately supplied to power amplifier outputs 3.4. 
Under the influence of these pulses emitting diodes 7.8 in the measurement and reference channels emit pulses of light. Through registers tokoreguliruyuschih 5, 6 regulated current flowing through the light emitting diodes 7.8, thereby changing the intensity and brightness of the light signals outputted from the light-emitting diodes 7.8.

Optical radiation through the first unbalanced coupler 9 to the first optical fiber sensor 14 is input to the second optical sensor 15 . In the absence of deformation of the solid structure 13 radiation intensity of the light coming through the second and fourth unbalanced connectors 11,12 to 19,20 photodetectors will be the same.

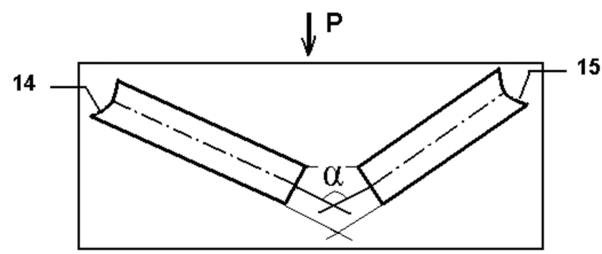

Light conductor 14. 15; aL -poteri in the measuring channel dB.

Figure 2. Shows the curve of Conditionally fibers under the influence of the load $P$ on a solid structure.

Operation is based on the change in light intensity sensor fibers under load R. When exposed to a solid structure is shifted ends of optical fibers 14 and 15 lying in one plane relative to each other, which leads to a reduction of the luminous flux intensity.

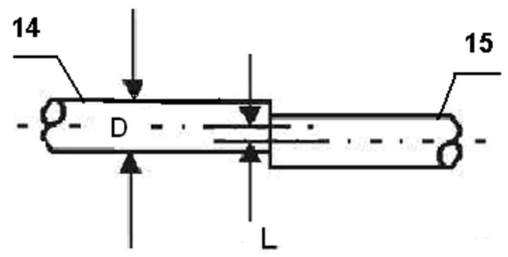

Figure 3. Conventionally, optical waveguides shown offset in the vertical shift of the solid-state design.

When the strain (or vertical displacement) solid structure 13 by the load $\mathrm{P}$ is shifted fibers 14,15 of the sensor with respect to each, other. The amount of luminous flux entering the first optical fiber 14 second optical fiber sensor 15 decreases, decreasing light intensity entering the second unbalanced connector 11. On its input will be the light signal corresponding to the difference in the measurement light signals and reference channels. Therefore electrical and the signal from the photodetector 19 will vary. The electrical signal from the photodetector 20 remains constant. The error signal from the photodetectors 19, 20 enters the photoelectric signal processing unit 21 , and then to the computer 22 . If the error signal in the measurement and reference channels is small, solid-state design is found fit, that is displayed on the monitor of the computer 22, and displayed the place a solid design 13 where the strain occurred.

When bending solid structure 13 (Figure 2) under a load loss $\mathrm{P}$ in the measuring channel defined by the formula will like

$$
a_{L}=-10 \lg \left[1-\frac{4 L}{\pi D}\right] \cos \alpha
$$

where $a_{L}-$ losses $d B$ in the measuring channel, L-offset between the centers of the optical fibers 14,15 sensor, mm, D-diameter optical fiber, $\mathrm{mm}, \alpha$-offset angle between the light guides 14, 15 of the sensor when exposed to load $\mathrm{P}$, $\pi$-constant.

Losses in the measurement channel light emission in the vertical displacement of the solid structure 13 by the action of the load $\mathrm{P}$ is given by:

$$
a_{L}=-10 \lg \left[1-\frac{4 L}{\pi D}\right]
$$

According to the relative ratio of the diameter D and the optical fibers shift along the characteristic L (Figure 4) obtained by experimentation can determine the loss of light radiation in the measuring channel, arising from the fact that not all the light flux from the first sensor fiber 14 enters the second light guide 15 sensor.

When you change the size of the gap between the light guides 14,15 on $\approx 0.01 \mathrm{~mm}$, measured deformation is from $1 * 10^{-4}$ to $2 * 10^{-2} \mathrm{~mm}$, the threshold sensitivity of the primary device is $1 * 10^{-4} \mathrm{~mm}$.

The clock is made on chip K155LAZ, trigger on a chip K155M2, photodetectors - on the photodiode VD25M, power amplifiers - the transistor KT315 unit photoelectric signal processing - on a chip K140UD1B, light-emitting diodes LED AL108A, fibers made of a polymer material.

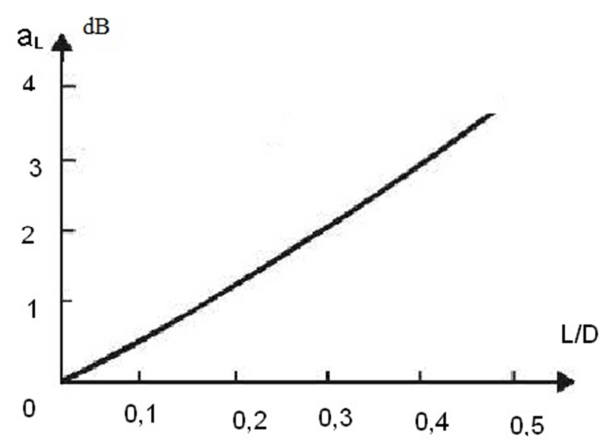

Figure 4. Shows static characteristic of the sensor device.

If necessary, the computer may be used instead of a measuring instrument for displaying the displacement L between the centers of the optical fibers in the measuring channel.

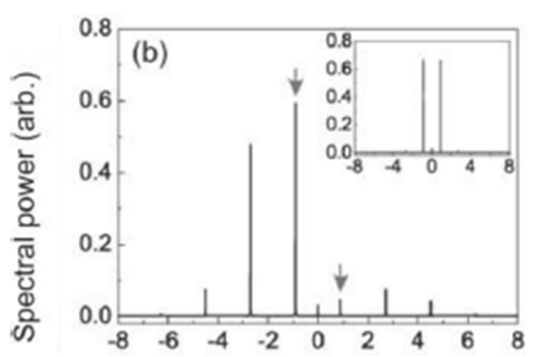

Figure 5. Only two-color light was launched with a frequency interval of $1,799 \mathrm{GHz}$. 


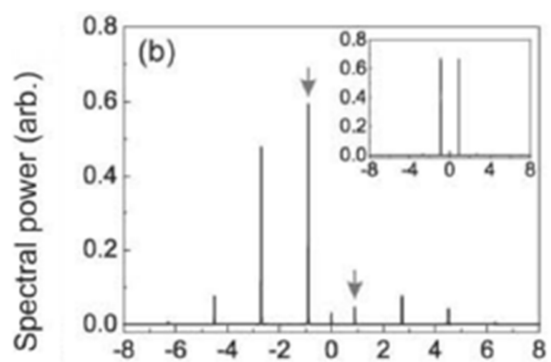

Figure 6. A tunable delayed train was launched together to amplify or suppress (insert) the scattering process.

Another method is to start a three-frequency laser pump with an adjustable phase ratio. Each of the two pairs of neighboring spectral components provides AR excitation. Both excitations interact coherently, as in the case of the first control procedure, and the scattering process can be amplified or suppressed, depending on their relative phases. In the experiment, we created a tricolor pump with equal optical power (280 $\mathrm{mW}$ at each frequency) using an electro-optical intensity modulator, controlling the relative phases for the adjustment of the bias point and the visibility of the modulation of the modulator. It is clear that the scattering process can be (Figure. 6) or suppressed (inset in Figure. 6).

The results show that AR can be optically controlled using pumping light with engineering amplitude envelope, spectrum and phase. The shown control methods can be used to implement optically controlled /switchable all-fiber acoustooptical devices, for example, frequency-crest generators and simulation elements in fiber lasers.

\section{Conclusion}

The proposed system is to use a specific pre-fracture and deformation of solid structures, in particular, building structures and hydraulic structures (dams). Since with the help of optical fibers can control the state of strain measurement structures at several points. Small, cheap and sensitive components for sensors that have low power consumption, high sensitivity and compliance with technical requirements can be effectively used to control various types of violations and mechanical damage. At present, thanks to new developments in solid state physics and fiber optics, significantly reducing the cost of optical fiber and semiconductor devices made available use of sensors based on optical fiber, not only enterprises, but also for non-destructive testing of buildings and other engineering structures, and their individual parts.

\section{References}

[1] BN Rakhimov, TD Rajabov. Optoelectronic system for detection of pre-destruction of objects and structures by means of optical fibers. Scientific and Technical Journal Vestnik TUIT, 2011 №2 C 35-39. Tashkent.

[2] Ovcharenko AB, Kuznetsov AS, Trushin V. D. Application of the OTDR in fiber optic systems for controlling the integrity of aircraft structures from composite materials / Proceedings of the VIII Symposium on Automation Problems in a Strength Experiment, Novosibirsk, 1990. - P. 109-112.

[3] BN Rakhimov, TD Rajabov. Optoelectronic monitoring system for composite building structures based on fiber-optic cables "Axborot texnologilari va telekom-munikatsiya muammolari" Republic of scientific and technical confrontation. 21-22 April 2011 in Tashkent.

[4] B. N. Rakhimov, T. D. Radjabov. Republican scientific and technical conference "Information technologies and telecommunication problems", which is a composite design of the optoeloconometers. April 21-22, 2011 Tashkent.

[5] Patent IAP 05166. BNRakhimov TDD. Radzhabov, D. A. Davronbekov, Z. T. Khakimov, A. Kurbanov. Device for the diagnosis of pre-destruction and deformation of solid-state structures from 19. 01. 16.

[6] Rakhimov N.R. Application of optical fiber in the system for assessing the fatigue damage of structural elements / Russia. Izv. universities. Instrument making, 2005. T. 48, No. 1. P. 39-43.

[7] Rakhimov N.R, Shamirzayev S.Kh. / Uzb. Physical Journal, 2004. T.6, No. 4. P. 288-293.

[8] B.N Rakhimov, E.Yu. Kutenkova, TVLarina, and OK Ushakov. The device and the method for determining the sites of pre-destruction of structures. Patent of the Russian Federation No. 2462698 .

[9] BN Rakhimov, TD Rajabov, D. A. Davronbekov, Z. T. Khakimov, A. Kurbanov. Optical sensor for the diagnosis of deformation of solid materials. Floor. decision on the application of the Patent of the Republic of Uzbekistan.

[10] Zak E.A. Fiber-optic converters with external modulation. M. Energoatomizdat, 1987, p. 11-15. 